\title{
How good are we at first-trimester prevention and prediction of early fetal growth restriction?
}

A series of recent publications have shown that early preeclampsia can be successfully predicted in the first trimester using a combination of maternal, biophysical and biochemical factors [1 -4], and administration of low-dose aspirin to women identified as high-risk can reduce the development of preterm preeclampsia by about $60 \%$ [5]. Given that abnormal placentation and placental insufficiency are commons pathway to many cases of preeclampsia and fetal growth restriction (FGR), we intuitively use the same strategies to predict and prevent FGR. However, are we as successful in FGR as we are in preeclampsia?

\section{A variety of definitions}

In contrast to preeclampsia, which is a clearly defined condition, multiple terms describe suboptimal fetal growth, and two of them, i.e. small for gestational age (SGA) and FGR, are often used interchangeably. This happens despite the two being two different conditions. SGA merely signifies a fetus (or a neonate) that is smaller than a given centile (usually the $10^{\text {th }}$ ), whereas FGR involves the failure of the fetus to reach its developmental potential, and it normally refers to a small fetus with some evidence of hypoxia. Therefore, one might say that FGR is a subset of SGA, and this is the case most of the times, but not always. A growth-restricted fetus can be non-SGA (i. e. $>10^{\text {th }}$ centile for estimated weight or abdominal circumference) and still be FGR, if its growth potential was meant to be higher. This reality has been acknowledged by the recent consensus definition for FGR, which introduces the option of a fetus crossing centiles, even if its eventual centile is $>10^{\text {th }}[6]$. Despite this development, different definitions have traditionally been used in the existing studies, even within the terms SGA and FGR (different cutoffs, Doppler parameters, pre- or postnatal weight etc), adding to the heterogeneity of the literature.

\section{Targeting small fetuses}

Prediction of FGR (or SGA) has been pursued along that of preeclampsia, and it was early acknowledged that the yield of screening models is higher when FGR coexists with preeclampsia rather than when it is an isolated condition. Four years ago, the Fetal Medicine Foundation (FMF) developed a dedicated screening algorithm targeting SCA, and they reported that, by combining maternal, biochemical and biophysical factors, about $50 \%$ of SGA can be predicted for a $10 \%$ false positive rate [7]. However, is this the case?

\section{Prediction of small fetuses through preeclampsia- oriented screening}

What happens in practice is that we screen for preeclampsia, we treat screen-positive cases aiming to prevent preeclampsia, and along the way we predict some of the FGR cases and prevent a subgroup of them. This is beautifully illustrated in a recent publication by the FMF, where data from the SPREE study (which is a screening study) were combined with data of the ASPRE trial (which is an interventional study) to show, among else, what happens with SGA when we screen and treat for preeclampsia. What this study found is that, by screening for preeclampsia, and using a cut-off of 1:100 for preeclampsia, we can predict $31 \%$ of neonates with birth weight $<10^{\text {th }}$ centile requiring delivery before 37 weeks, and $35 \%$ of such babies requiring delivery before 32 weeks [1]. Of course, the prediction rates significantly increased for small neonates with coexisting preeclampsia, but this is a different group whatsoever. Focusing on even smaller neonates (birthweight $<3^{\text {rd }}$ centile) in the absence of preeclampsia, this screening strategy can predict about $40 \%$ of those requiring delivery before 37 or 32 weeks [1]. So, in practice, screening for preeclampsia with a $10 \%$ screen-positive rate (which is where 1:100 corresponds to) can predict about $35-40 \%$ of the small and very small fetuses requiring early delivery.

\section{Prevention of small fetuses through preeclampsia- oriented screening}

Administration of prophylactic aspirin to these screen-positive (for preeclampsia) cases, will significantly reduce the risk for small neonates with preeclampsia, but will only reduce the risk for the subgroup of such neonates without preeclampsia that require delivery before 32 weeks, by about $60 \%$. Of course this is quite an important group in terms of morbidity, but it only comprises about $0.25 \%$ of the population [1].

\section{A synopsis...}

It appears thus that combined first-trimester screening for preeclampsia can predict about $40 \%$ of the subgroup of small fetuses who will need a preterm delivery. Prophylactic aspirin in screen-positive cases will reduce the risk for the small but clinically important group of very preterm (<32 weeks) small fetuses by about $60 \%$. 


\section{...and some more \\ unanswered questions}

There are yet more unanswered questions, including the possible role of maternal hemodynamics as part of a combined screening, or the ability of the fetal fraction of cell-free DNA in the maternal blood to act as an independent predictor. And, of course, there is the issue with twins. There is an ongoing debate whether twin-specific growth charts should be used, or charts of singleton are applicable as well, so problems like this need to be solved before aiming at screening.

\section{Dr Alexandros Sotiriadis}

Dr Konstantinos Dinas

Second Department of Obstetrics and Gynecology

Faculty of Medicine, Aristotle University of Thessaloniki, Greece

\section{References}

[1] Tan MY, Poon LC, Rolnik DL et al. Prediction and prevention of small-for-gestational-age neonates: evidence from SPREE and ASPRE. Ultrasound Obstet Gynecol 2018

[2] Tan MY, Wright D, Syngelaki A et al. Comparison of diagnostic accuracy of early screening for pre-eclampsia by NICE guidelines and a method combining maternal factors and biomarkers: results of SPREE. Ultrasound Obstet Gynecol 2018

[3] O'Gorman N, Wright D, Poon LC et al. Multicenter screening for preeclampsia by maternal factors and biomarkers at 11-13 weeks' gestation: comparison to NICE guidelines and ACOG recommendations. Ultrasound Obstet Gynecol 2017

[4] O'Gorman N, Wright D, Poon LC et al. Accuracy of competing risks model in screening for pre-eclampsia by maternal factors and biomarkers at 11-13 weeks' gestation. Ultrasound Obstet Gynecol 2017
[5] Rolnik DL, Wright D, Poon LC et al. Aspirin versus Placebo in Pregnancies at High Risk for Preterm Preeclampsia. N Engl J Med 2017

[6] Gordijn S], Beune IM, Thilaganathan B et al. Consensus definition of fetal growth restriction: a Delphi procedure. Ultrasound Obstet Gynecol 2016; 48: 333 -339

[7] Poon LC, Syngelaki A, Akolekar R et al. Combined screening for preeclampsia and small for gestational age at 11-13 weeks. Fetal Diagn Ther 2013; 33: 16-27 